\section{Beam Skirt Effects When Doing EDS In An Low-Vacuum SEM}

J.B. Blide-Soerensen

Risoe National Laboratory, Denmark

Approximately $75 \%$ of the primary electrons will be scattered when you use a working distance of $12 \mathrm{~mm}$ and a pressure of 3.5 torr, and a significant fraction of these scattered electrons will hit the sample farther away from the beam target than $50 \mu \mathrm{m}$. (The scattered intensity is approximately given by $\mid s / l_{0}=1-\exp (-\rho \sigma L / k T)$ where $\rho$ is the pressure, $\sigma$ the total scattering cross section for electron scattering on the gas used, $L$ the distance between the last pressure limiting aperture and the sample. $k$ the Boltzmann constant and $T$ the absolute temperature).

Examples of skirt shapes are e.g... given in:

D. A. Moncrieff et al., J. Phys. D: Appl. Phys. vol. 12 (1979) 481-88.

D. C. Joy, Microscopy and Microanalysis ' 96 , Proc. Annual Meeting MSA, Minneapolis, Minnesota, 11 -15 August 1996

It is to a large degree possible to correct for the beam skirt effects:

1) You can extrapolate from spectral measurements made at several different pressures to the result that would have been found without scattering, provided that the measurements are made in the single scattering regime (i.e., $\mathrm{pL}<$ approximately $1.6 \mathrm{~Pa}^{*} \mathrm{~m}$ for measurements in water vapour). You can do this because the beam shape to a good approximation is independent of pressure in the single scattering regime, so that only the skirt intensity is changing with pressure. The extrapolation is made with use of the equation:

$\mathrm{C}_{T}=\mathrm{Cu}_{u} \cdot \exp (-p \sigma L / k T)+\mathrm{C}_{\mathrm{S}} \cdot(1-\exp (-\rho \sigma L / k T))$

which gives $C_{T}=\left(C_{U}-C_{S}\right) \cdot \exp (-\rho \sigma L / k T)+C_{S}$ where $C_{T}$ is the actual count rate at pressure $p$ for the $X$-ray peak of interest, $C_{U}$ is the count rate that would have been obtained if no electrons had been scattered and $\mathrm{C}_{\mathrm{s}}$ is the count rate that would have been obtained if all electrons were scattered once. The best results are obtained if the extrapolation is made by a weighted least squares fitting. In order to obtain single scattering conditions, you can use a so called $X$-ray bullet to reduce the working distance.

2) If there is plural scattering, you can take two spectra, one with a fine needle (of the kind used for field ion microscopy or scanning tunneling microscopy) inserted over the point of interest, and the other with the needle slightly $\frac{5}{5}$ retracted. The characteristic peaks from the needle are removed from the spec- $\frac{\bar{n}}{\vec{D}}$ tra. Subtraction of the first from the second spectrum will now approximately give ㅇ the spectrum from the point of interest.

Neither method will give you as exact an analysis as you will get under high vacuum, but you can get rid of most of the skirt effects. The pressure variation $\frac{0}{3}$ method in particular yields pretty good results if carefully performed. The meth- 흠 ods are described in:

J. B. Blide-Soerensen and C. C. Appel, Proc. 48th Annual Meeting of the Scandinavian Society for Electron Microscopy, Aarhus, 2 - 5 June 1996, pp. 4 - 5.

J. B. Bilde-Soerensen and C. C. Appel, Proc. 11th European Congress on Microscopy EUREM '96, Dublin, 26-30 August 1996. Session T6.

J. B. Bilde-Soerensen and C. C. Appel, Proc. 49th Meeting Annual of the Scandinavian Society for Electron Microscopy.. Gothenburg, 10 - 13 June 1997, pp. 12-15.

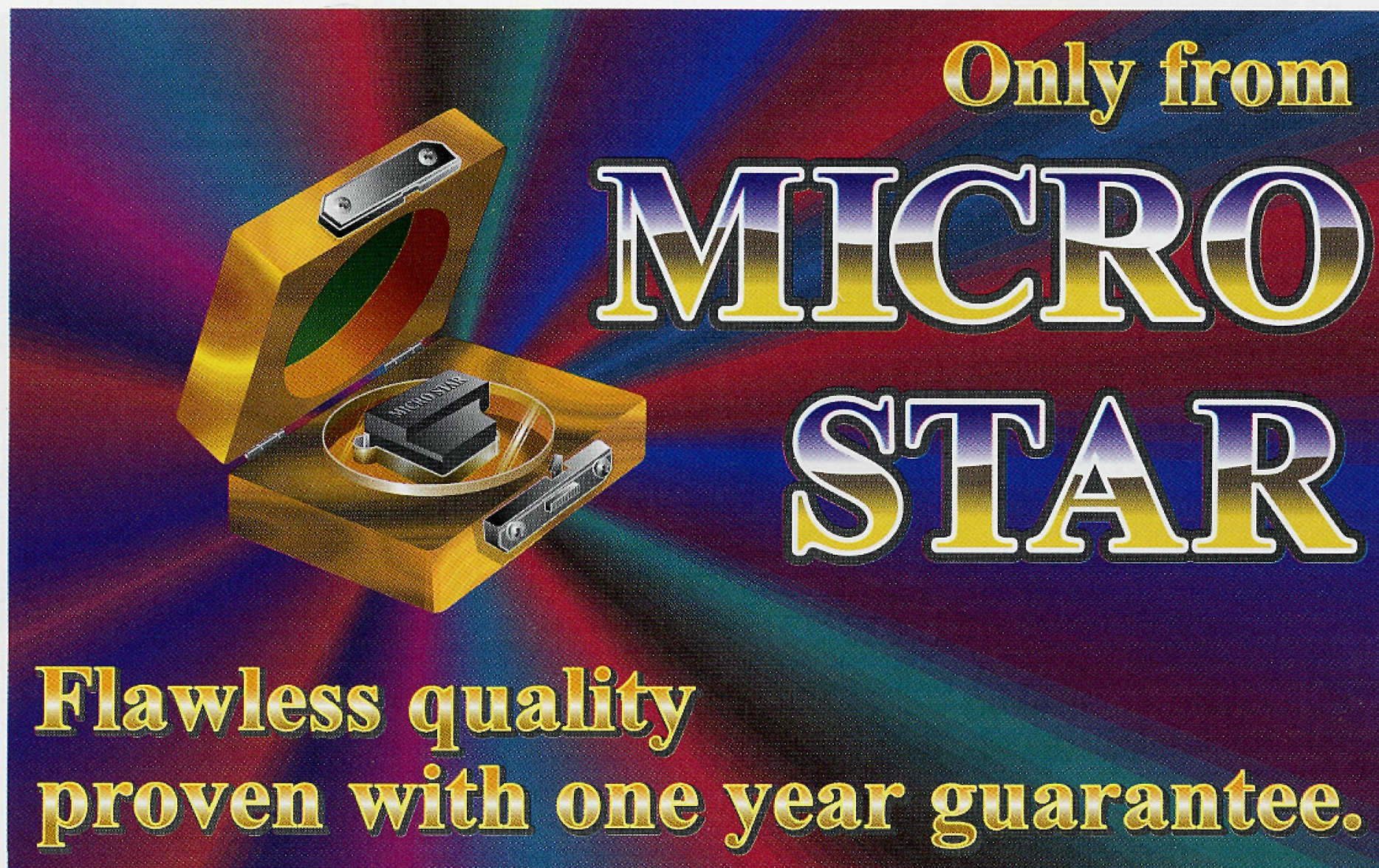

MICRO STAR DIAMOND KNIVES 8005332509 FAX 4092949861 E-MAIL MISTAR@ MSN.COM 


\section{A Cut Above the Rest ...}

\section{Choice of Computer Platform}

\section{Digital Detectors with 115eV Resolution}

For over thirty years, Princeton Gamma-Tech has been the forerunner in $X$-ray Micr oanalysis and Computer-Aided Imaging. The PGT IMIX pr ovides today's microscopist a comprehensive suite of easy to use tools that make it easier to acquire and analyze data in less time. Among these is PGT's Position-Tagged Spectrometry, where in one simple near frame-rate collection, you have all the sample information you will ever need. PGT also offers a full line of award winning PRISM Digital Detectors. With our
patented digital acquisition, PRISM offers superior light element
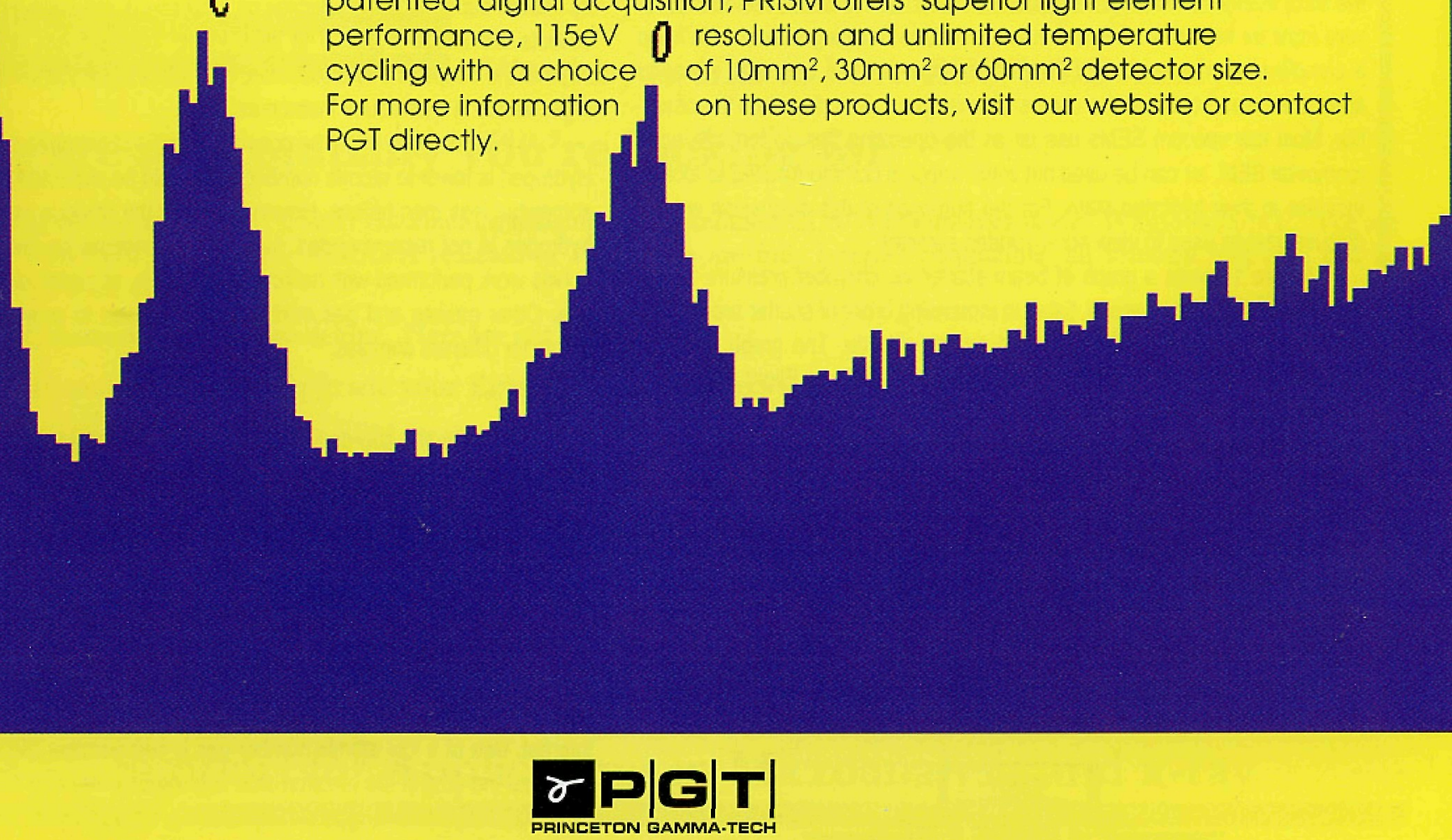

Princeton Gamma-Tech, Inc. C/N 863 Princeton, NJ 08542-0863

Telephone: $609 \cdot 924 \cdot 7310$ • Facsimile: 609•924-1729 • Website: www.pgt.com • e-mail: sales@pgt.com 